\title{
A partially parallel-in-time fixed-stress splitting method for Biot's consolidation model
}

\author{
Manuel Borregales a , Kundan Kumar a , Florin Adrian Radu a , Carmen Rodrigo c,*, \\ Francisco José Gaspar ${ }^{\mathrm{b}}$ \\ ${ }^{a}$ Department of Mathematics, University of Bergen, Allégaten 41, 50520 Bergen, Norway

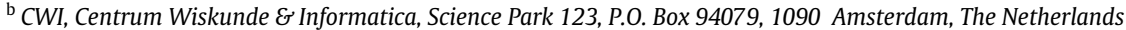 \\ ' IUMA and Department of Applied Mathematics, University of Zaragoza, María de Luna, 3, 50018 Zaragoza, Spain
}

\section{A R T I C L E I N F O}

\section{Article history:}

Available online $\mathrm{xxxx}$

\section{Keywords:}

Iterative fixed-stress splitting scheme

Parallel-in-time

Biot's model

Poromechanics

Finite elements

Convergence analysis

\begin{abstract}
A B S T R A C T
In this work, we study the parallel-in-time iterative solution of coupled flow and geomechanics in porous media, modelled by a two-field formulation of Biot's equations. In particular, we propose a new version of the fixed-stress splitting method, which has been widely used as solution method of these problems. This new approach forgets about the sequential nature of the temporal variable and considers the time direction as a further direction for parallelization. The method is partially parallel-in-time. We present a rigorous convergence analysis of the method and numerical experiments to demonstrate the robust behaviour of the algorithm.
\end{abstract}

(c) 2018 Elsevier Ltd. All rights reserved.

\section{Introduction}

The coupled poroelastic equations describe the behaviour of fluid-saturated porous materials undergoing deformation. Such coupling has been intensively investigated, starting from the pioneering one-dimensional work of Terzaghi [1], which was extended to a more general three-dimensional theory by Biot [2,3]. Biot's model was originally developed to study geophysical applications such as reservoir geomechanics, however, nowadays it is widely used in the modelling of many applications in a great variety of fields, ranging from geomechanics and petroleum engineering, to biomechanics or food processing. There is a vast literature on Biot's equations and the existence, uniqueness, and regularity of their solutions, see Showalter [4], Phillips and Wheeler [5] and the references therein.

Reliable numerical methods for solving poroelastic problems are needed for the accurate solution of multi-physics phenomena appearing in different application areas. In particular, the solution of the large linear systems of equations arising from the discretization of Biot's model is the most consuming part when real simulations are performed. For this reason, a lot of effort has been made in the last years to design efficient solution methods for these problems. Two different approaches can be adopted, the so-called monolithic or fully coupled methods and the iterative coupling methods. The monolithic approach consists of solving the linear system simultaneously for all the unknowns. The challenge here, is the design of efficient preconditioners to accelerate the convergence of Krylov subspace methods and the design of efficient smoothers in a multigrid framework. Recent advances in both directions can be found in [6-9] and the references therein. These methods usually provide unconditional stability and convergence. Iterative coupling methods, however, solve sequentially the equations for fluid flow and geomechanics, at each time step, until a converged solution within a prescribed tolerance is

\footnotetext{
* Corresponding author.

E-mail addresses: Manuel.Borregales@uib.no (M. Borregales), Kundan.Kumar@uib.no (K. Kumar), Florin.Radu@uib.no (F.A. Radu), carmenr@unizar.es (C. Rodrigo), F.J.Gaspar@cwi.nl (F.J. Gaspar).
} 
achieved. They offer several attractive features as their flexibility, for example, since they allow to link two different codes for fluid flow and geomechanics for solving the coupled poroelastic problems. The most used iterative coupling methods are the drained and undrained splits, which solve the mechanical problem first, and the fixed-strain and fixed-stress splits, which on the contrary solve the flow problem first [10-12].

Among iterative coupling schemes, the fixed-stress splitting method is the most widely used. This sequential-implicit method basically consists of solving the flow problem first fixing the volumetric mean total stress, and then the mechanics part is solved from the values obtained at the previous flow step. In the last years, a lot of research has been done on this method. The unconditional stability of the fixed-stress splitting method is shown in [11] using a von Neumann analysis. In addition, stability and convergence of the fixed-stress splitting method have been rigorously established in [13]. Recently, in [14] the authors have proven the convergence of the fixed-stress split method in energy norm for heterogeneous problems. Estimates for the case of the multirate iterative coupling scheme are obtained in [15], where multiple finer time steps for flow are taken within one coarse mechanics time step, exploiting the different time scales for the mechanics and flow problems. In [16], the convergence of this method is demonstrated in the fully discrete case when space-time finite element methods are used. In [17], the authors present a very interesting approach which consists of re-interpreting the fixed-stress splitting scheme as a preconditioned-Richardson iteration with a particular block-triangular preconditioning operator. Recently, in [18] an inexact version of the fixed-stress splitting scheme has been successfully proposed as smoother in a geometric multigrid framework, which provides an efficient monolithic solver for Biot's problem. Finally, we mention that the fixedstress splitting was recently applied to a non-linear poromechanics model in [19] and to consolidation of unsaturated porous media in [20].

All the previously mentioned algorithms are based on a time-marching approach, in which each time step is solved after the other in a sequential manner, and therefore they do not allow the parallelization of the temporal variable. Parallel-in-time integration methods, however, are receiving a lot of interest nowadays because of the advent of massively parallel systems with thousands of threads, permitting to reduce drastically the computing time [21]. There are various different methods introducing concurrency along the temporal dimension. The most well-known time-parallelization methods include the parallel full approximation scheme in space and time (PFASST) [22], the Parareal method [23], the Multigrid Reduction in Time algorithm (MGRIT) [24], the Space-time Multigrid method (STMG) [25], and the Space-time concurrent multigrid waveform relaxation (WRMG) with cyclic reduction [26,27]. Due to the mixed elliptic-parabolic structure of Biot's problem, the development of parallel-in-time algorithms is not intuitive.

In the present work, we introduce a very simple version of the fixed-stress splitting method for the poroelasticity problem which is partially parallel-in-time. We further show rigorously its convergence. Techniques similar with the ones from $[13,14,16]$ are used. For completeness, in Section 3, we include a new proof for the convergence of the fixed-stress splitting algorithm in the semi-discrete case. The theoretical results are sustained by numerical computations. Moreover, a fully parallel-in-time version of the presented method is introduced.

The remainder of the paper is organized as follows. In Section 2 we briefly introduce the poroelasticity model and present the considered finite element discretizations. Section 3 is devoted to the description of the classical fixed-stress splitting algorithm. In Section 4, the partially parallel-in-time new approach based on the fixed-stress splitting algorithm is presented and its convergence analysis is derived. Section 5 illustrates the robustness of the proposed parallel-in-time fixed-stress splitting method through two numerical experiments. Finally, some conclusions are drawn in Section 6.

\section{Mathematical model and discretization}

The equations describing poroelastic flow and deformation are derived from the principles of fluid mass conservation and the balance of forces on the porous matrix. More concretely, according to Biot's theory [2,3], and assuming $\Omega$ a bounded open subset of $\mathbb{R}^{d}, d \in\{2,3\}$, with regular boundary $\Gamma$, the consolidation process must satisfy on the space-time domain $\Omega \times(0, T]$ the following system of partial differential equations:

equilibrium equation:

constitutive equation:

compatibility condition:

Darcy's law:

continuity equation:

$$
\begin{aligned}
-\operatorname{div} \boldsymbol{\sigma}^{\prime}+\alpha \nabla p & =\rho \mathbf{g}, \\
\boldsymbol{\sigma}^{\prime} & =2 G \boldsymbol{\varepsilon}(\boldsymbol{u})+\lambda \operatorname{div}(\boldsymbol{u}) \boldsymbol{I}, \\
\boldsymbol{\varepsilon}(\boldsymbol{u}) & =\frac{1}{2}\left(\nabla \boldsymbol{u}+\nabla \boldsymbol{u}^{t}\right), \\
\mathbf{q} & =-\frac{1}{\mu_{f}} \boldsymbol{K}\left(\nabla p-\rho_{f} \mathbf{g}\right),
\end{aligned}
$$$$
\frac{\partial}{\partial t}\left(\frac{1}{\beta} p+\alpha \nabla \cdot \boldsymbol{u}\right)+\nabla \cdot \boldsymbol{q}=f,
$$

where $\boldsymbol{I}$ is the identity tensor, $\boldsymbol{u}$ is the displacement vector, $p$ is the pore pressure, $\boldsymbol{\sigma}^{\prime}$ and $\boldsymbol{\varepsilon}$ are the effective stress and strain tensors for the porous medium, $\boldsymbol{g}$ is the gravity vector, $\boldsymbol{q}$ is the percolation velocity of the fluid relative to the soil, $\mu_{f}$ is the fluid viscosity and $\boldsymbol{K}$ is the absolute permeability tensor. The Lamé coefficients, $\lambda$ and $G$, can be also expressed in terms of Young's modulus $E$ and the Poisson's ratio $v$ as $\lambda=E v /((1-2 v)(1+v))$ and $G=E /(2+2 v)$. The bulk density $\rho$ is related to the densities of the solid $\left(\rho_{s}\right)$ and fluid $\left(\rho_{f}\right)$ phases as $\rho=\phi \rho_{f}+(1-\phi) \rho_{s}$, where $\phi$ is the porosity. $\beta$ is the Biot modulus and $\alpha$ is the Biot coefficient given by $\alpha=1-K_{b} / K_{s}$, where $K_{b}$ is the drained bulk modulus, and $K_{s}$ is the bulk modulus of the solid phase. 
If considering the displacements of the solid matrix $\boldsymbol{u}$ and the pressure of the fluid $p$ as primary variables, we obtain the so-called two-field formulation of the Biot's consolidation model. With this idea in mind, the mathematical model (1) can be rewritten as

$$
\begin{gathered}
-\operatorname{div} \boldsymbol{\sigma}^{\prime}+\alpha \nabla p=\rho \mathbf{g}, \quad \boldsymbol{\sigma}^{\prime}=2 G \boldsymbol{\varepsilon}(\boldsymbol{u})+\lambda \operatorname{div}(\boldsymbol{u}) \boldsymbol{I}, \\
\frac{\partial}{\partial t}\left(\frac{1}{\beta} p+\alpha \nabla \cdot \boldsymbol{u}\right)-\nabla \cdot\left(\frac{1}{\mu_{f}} \boldsymbol{K}\left(\nabla p-\rho_{f} \boldsymbol{g}\right)\right)=f .
\end{gathered}
$$

The most important feature of this mathematical model is that the equations are strongly coupled. Here, the Biot parameter $\alpha$ plays the role of coupling parameter between these equations. In order to ensure the existence and uniqueness of solution, we must supplement the system with appropriate boundary and initial conditions. For instance,

$$
\boldsymbol{u}=\mathbf{0}, \quad \text { on } \Gamma_{\mathbf{u}} \text { and } \quad \boldsymbol{\sigma}^{\prime} \boldsymbol{n}=\mathbf{0}, \quad \text { on } \Gamma_{t},
$$

where $\boldsymbol{n}$ is the unit outward normal to the boundary, $\Gamma_{p} \cup \Gamma_{\mathbf{q}}=\Gamma_{t} \cup \Gamma_{\mathbf{u}}=\Gamma$, and $\Gamma_{p} \cap \Gamma_{\mathbf{q}}=\Gamma_{t} \cap \Gamma_{\mathbf{u}}=\emptyset$ with $\Gamma_{p}, \Gamma_{\mathbf{q}}, \Gamma_{\mathbf{u}}$ and $\Gamma_{t}$ subsets of $\Gamma$ having non null measure. For the initial time, $t=0$, the following condition is fulfilled

$$
\left(\frac{1}{\beta} p+\alpha \nabla \cdot \boldsymbol{u}\right)(\boldsymbol{x}, 0)=0, \boldsymbol{x} \in \Omega .
$$

Results about existence and uniqueness of the solution of the Biot's model (2)-(3) with initial condition (5) can be found in the works by Showalter [4] and Zenisek [28].

\subsection{Semi-discretization in space}

To introduce the spatial discretization of the Biot model, we choose the finite element method. We define the standard Sobolev spaces $\boldsymbol{V}=\left\{\boldsymbol{u} \in\left(H^{1}(\Omega)\right)^{d}|\boldsymbol{u}|_{\Gamma_{\mathbf{u}}}=\mathbf{0}\right\}$, and $Q=\left\{p \in H^{1}(\Omega)|p|_{\Gamma_{p}}=0\right\}$, with $H^{1}(\Omega)$ denoting the Hilbert subspace of $L_{2}(\Omega)$ of functions with first weak derivatives in $L_{2}(\Omega)$. Then, we introduce the variational formulation for the two-field formulation of the Biot's model as follows: Find $(\boldsymbol{u}(t), p(t)) \in C^{1}([0, T] ; \boldsymbol{V}) \times C^{1}([0, T] ; Q)$ such that

$$
\begin{aligned}
& a(\boldsymbol{u}(t), \boldsymbol{v})-\alpha(p(t), \operatorname{div} \boldsymbol{v})=(\rho \mathbf{g}, \boldsymbol{v}), \forall \boldsymbol{v} \in \boldsymbol{V}, t \in(0, T], \\
& \alpha\left(\operatorname{div} \partial_{t} \boldsymbol{u}(t), q\right)+\frac{1}{\beta}\left(\partial_{t} p(t), q\right)+b(p(t), q)=(f, q) \\
& +\left(\boldsymbol{K} \mu_{f}^{-1} \rho_{f} \mathbf{g}, \nabla q\right), \forall q \in Q, t \in(0, T],
\end{aligned}
$$

where $(\cdot, \cdot)$ is the standard inner product in the space $L_{2}(\Omega)$, and the bilinear forms $a(\cdot, \cdot)$ and $b(\cdot, \cdot)$ are given as

$$
\begin{aligned}
a(\boldsymbol{u}, \boldsymbol{v}) & =2 G \int_{\Omega} \boldsymbol{\varepsilon}(\boldsymbol{u}): \boldsymbol{\varepsilon}(\boldsymbol{v}) d \Omega+\lambda \int_{\Omega} \operatorname{div} \boldsymbol{u} \operatorname{div} \boldsymbol{v} d \Omega, \\
b(p, q) & =\int_{\Omega} \frac{\boldsymbol{K}}{\mu_{f}} \nabla p \cdot \nabla q d \Omega .
\end{aligned}
$$

Finally, the initial condition is given by

$$
\left(\frac{1}{\beta} p(0)+\alpha \nabla \cdot \boldsymbol{u}(0), q\right)=0, \quad \forall q \in L_{2}(\Omega) .
$$

It is important to consider a finite element pair of spaces $\boldsymbol{V}_{h} \times Q_{h}$ satisfying an inf-sup condition. One very simple choice would be the stabilized P1-P1 scheme firstly introduced in [29] and widely analysed in [30], in which $\boldsymbol{V}_{h}$ consists of the space of piecewise (with respect to a triangulation $\mathcal{T}_{h}$ ) linear continuous vector valued functions on $\Omega$ and the space $Q_{h}$ consists of piecewise linear continuous scalar valued functions. Other choices would be P2-P1, that is, piecewise quadratic continuous vector valued functions for displacements and piecewise linear continuous scalar valued functions for pressure, widely studied by Murad and Loula [31-33]; or the so-called MINI element [30] in which $\boldsymbol{V}_{h}=\boldsymbol{V}_{l} \oplus \boldsymbol{V}_{b}$, where $\boldsymbol{V}_{l}$ is the space of piecewise linear continuous vector valued functions and $\boldsymbol{V}_{b}$ is the space of bubble functions. Discrete inf-sup stability conditions and convergence results for the stabilized P1-P1 and the MINI element were recently derived in [30].

The semi-discretized problem can be written as follows: Find $\left(\boldsymbol{u}_{h}(t), p_{h}(t)\right) \in C^{1}\left([0, T] ; \boldsymbol{V}_{h}\right) \times C^{1}\left([0, T] ; Q_{h}\right)$ such that

$$
\begin{aligned}
& \begin{array}{l}
a\left(\boldsymbol{u}_{h}(t), \boldsymbol{v}_{h}\right)-\alpha\left(p_{h}(t), \operatorname{div} \boldsymbol{v}_{h}\right)=\left(\rho \mathbf{g}, \boldsymbol{v}_{h}\right), \forall \boldsymbol{v}_{h} \in \boldsymbol{V}_{h}, t \in(0, T], \\
\alpha\left(\operatorname{div} \partial_{t} \boldsymbol{u}_{h}(t), q_{h}\right)+\frac{1}{\beta}\left(\partial_{t} p_{h}(t), q_{h}\right)+b\left(p_{h}(t), q_{h}\right)=\left(f_{h}, q_{h}\right) \\
+\left(\boldsymbol{K} \mu_{f}^{-1} \rho_{f} \mathbf{g}, \nabla q_{h}\right), \forall q_{h} \in Q_{h}, t \in(0, T],
\end{array}
\end{aligned}
$$


giving rise to the following fully coupled algebraic/differential equations system,

$$
\left[\begin{array}{cc}
0 & 0 \\
B & M_{p}
\end{array}\right]\left[\begin{array}{c}
\dot{u}_{h} \\
\dot{p}_{h}
\end{array}\right]+\left[\begin{array}{cc}
A & B^{t} \\
0 & -C
\end{array}\right]\left[\begin{array}{c}
u_{h} \\
p_{h}
\end{array}\right]=\left[\begin{array}{l}
g_{h} \\
\widetilde{f}_{h}
\end{array}\right],
$$

where we have denoted $\dot{u}_{h} \equiv \partial_{t} \boldsymbol{u}_{h}(t)$ and $\dot{p}_{h} \equiv \partial_{t} p_{h}(t)$.

Remark 1. We wish to emphasize that the solver based on the fixed-stress splitting method, which we are going to propose in this work, can be applied to other different discretizations of the problem, for example, mixed finite-elements or finite volume schemes.

\section{The fixed-stress splitting algorithm for the semi-discretized problem}

A popular alternative for solving the poroelasticity problem in an iterative manner is the so-called fixed-stress splitting method. This scheme is based on solving the flow equation by adding the stabilization term $L \frac{\partial p}{\partial t}$ on both sides of the equation:

$$
\left(\frac{1}{\beta}+L\right) \frac{\partial p}{\partial t}-\nabla \cdot\left(\frac{1}{\mu_{f}} \boldsymbol{K}\left(\nabla p-\rho_{f} \mathbf{g}\right)\right)=f-\alpha \frac{\partial}{\partial t}(\nabla \cdot \mathbf{u})+L \frac{\partial p}{\partial t},
$$

where $L$ is a parameter to fix, and then, the mechanics problem is solved using updated pressure. For more details about the algorithm and how to fix parameter $L$, see $[11,13,14,16]$. Thus, given an initial guess $\left(\boldsymbol{u}_{h}^{0}(t), p_{h}^{0}(t)\right)$, the fixed-stress splitting algorithm gives us a sequence of approximations $\left(\boldsymbol{u}_{h}^{i}(t), p_{h}^{i}(t)\right), i \geq 1$ as follows:

Step 1: Given $\left(\boldsymbol{u}_{h}^{i-1}(t), p_{h}^{i-1}(t)\right) \in C^{1}\left([0, T] ; \boldsymbol{V}_{h}\right) \times C^{1}\left([0, T] ; Q_{h}\right)$, find $p_{h}^{i}(t) \in C^{1}\left([0, T] ; Q_{h}\right)$ such that

$$
\begin{aligned}
& \left(\frac{1}{\beta}+L\right)\left(\partial_{t} p_{h}^{i}(t), q_{h}\right)+b\left(p_{h}^{i}(t), q_{h}\right)+\alpha\left(\operatorname{div} \partial_{t} \boldsymbol{u}_{h}^{i-1}(t), q_{h}\right)=L\left(\partial_{t} p_{h}^{i-1}(t), q_{h}\right)+ \\
& \quad\left(f_{h}, q_{h}\right)+\left(\boldsymbol{K} \mu_{f}^{-1} \rho_{f} \mathbf{g}, \nabla q_{h}\right), \quad \forall q_{h} \in Q_{h}, t \in(0, T], \text { and } \\
& p_{h}^{i}(0)=p_{0} .
\end{aligned}
$$

Step 2: Given $p_{h}^{i}(t) \in C^{1}\left([0, T] ; Q_{h}\right)$, find $\boldsymbol{u}_{h}^{i}(t) \in C^{1}\left([0, T] ; \boldsymbol{V}_{h}\right)$ such that

$$
a\left(\boldsymbol{u}_{h}^{i}(t), \boldsymbol{v}_{h}\right)=\alpha\left(p_{h}^{i}(t), \operatorname{div} \boldsymbol{v}_{h}\right)+\left(\rho \mathbf{g}, \boldsymbol{v}_{h}\right), \quad \forall \boldsymbol{v}_{h} \in \boldsymbol{V}_{h}, t \in(0, T] .
$$

The algorithm starts with an initial approximation $\left(\boldsymbol{u}_{h}^{0}(t), p_{h}^{0}(t)\right)$ defined along the whole time-interval. A natural choice is to take this approximation constant and equal to the values specified by the initial condition, $\left(\boldsymbol{u}_{h}^{0}(t), p_{h}^{0}(t)\right)=\left(\boldsymbol{u}_{0}, p_{0}\right), t \in$ $(0, T]$.

\subsection{Convergence analysis in the semi-discrete case}

Let $\delta \boldsymbol{u}_{h}^{i}(t)=\boldsymbol{u}_{h}^{i}(t)-\boldsymbol{u}_{h}^{i-1}(t)$ and $\delta p_{h}^{i}(t)=p_{h}^{i}(t)-p_{h}^{i-1}(t)$ denote the difference between two successive approximations for displacements and for pressure, respectively.

Theorem 1. The fixed-stress splitting method given in (13)-(14) converges for any $L \geq \frac{\alpha^{2}}{2\left(\frac{2 G}{d}+\lambda\right)}$. There holds

$$
\int_{0}^{t}\left\|\partial_{t} \delta p_{h}^{i}(s)\right\|^{2} d s \leq \frac{L}{\left(\frac{1}{\beta}+L\right)} \int_{0}^{t}\left\|\partial_{t} \delta p_{h}^{i-1}(s)\right\|^{2} d s .
$$

Proof. We take the time derivative of the difference of two successive iterates of the mechanics equation (14) and test the resulting equation by $\mathbf{v}_{h}=\partial_{t} \delta \mathbf{u}_{h}^{i-1}$ to get

$$
2 G\left(\boldsymbol{\varepsilon}\left(\partial_{t} \delta \mathbf{u}_{h}^{i}\right), \boldsymbol{\varepsilon}\left(\partial_{t} \delta \mathbf{u}_{h}^{i-1}\right)\right)+\lambda\left(\nabla \cdot \partial_{t} \delta \mathbf{u}_{h}^{i}, \nabla \cdot \partial_{t} \delta \mathbf{u}_{h}^{i-1}\right)-\alpha\left(\partial_{t} \delta p_{h}^{i}, \nabla \cdot \partial_{t} \delta \mathbf{u}_{h}^{i-1}\right)=0 .
$$

By taking the difference between two successive iterates of the flow Eq. (13) and testing with $q_{h}=\partial_{t} \delta p_{h}^{i}$, we obtain

$$
\frac{1}{\beta}\left\|\partial_{t} \delta p_{h}^{i}\right\|^{2}+L\left(\partial_{t}\left(\delta p_{h}^{i}-\delta p_{h}^{i-1}\right), \partial_{t} \delta p_{h}^{i}\right)+b\left(\delta p_{h}^{i}, \partial_{t} \delta p_{h}^{i}\right)+\alpha\left(\nabla \cdot \partial_{t} \delta \mathbf{u}_{h}^{i-1}, \partial_{t} \delta p_{h}^{i}\right)=0 .
$$

After summing up Eqs. (16) and (17), and using the identities

$$
(\sigma, \xi)=\frac{1}{4}\|\sigma+\xi\|^{2}-\frac{1}{4}\|\sigma-\xi\|^{2}, \quad(\sigma-\xi, \sigma)=\frac{1}{2}\left(\|\sigma\|^{2}-\|\xi\|^{2}+\|\sigma-\xi\|^{2}\right),
$$

one has

$$
\frac{G}{2}\left\|\boldsymbol{\varepsilon}\left(\partial_{t} \delta \mathbf{u}_{h}^{i}+\partial_{t} \delta \mathbf{u}_{h}^{i-1}\right)\right\|^{2}+\frac{\lambda}{4}\left\|\nabla \cdot\left(\partial_{t} \delta \mathbf{u}_{h}^{i}+\partial_{t} \delta \mathbf{u}_{h}^{i-1}\right)\right\|^{2}+\frac{1}{\beta}\left\|\partial_{t} \delta p_{h}^{i}\right\|^{2}
$$




$$
\begin{aligned}
& +\frac{1}{2} \frac{d}{d t}\left\|\delta p_{h}^{i}\right\|_{B}^{2}+\frac{L}{2}\left(\left\|\partial_{t} \delta p_{h}^{i}\right\|^{2}-\left\|\partial_{t} \delta p_{h}^{i-1}\right\|^{2}+\left\|\partial_{t} \delta p_{h}^{i}-\partial_{t} \delta p_{h}^{i-1}\right\|^{2}\right) \\
& =\frac{G}{2}\left\|\boldsymbol{\varepsilon}\left(\partial_{t} \delta \mathbf{u}_{h}^{i}-\partial_{t} \delta \mathbf{u}_{h}^{i-1}\right)\right\|^{2}+\frac{\lambda}{4}\left\|\nabla \cdot\left(\partial_{t} \delta \mathbf{u}_{h}^{i}-\partial_{t} \delta \mathbf{u}_{h}^{i-1}\right)\right\|^{2} .
\end{aligned}
$$

Next, we consider the time derivative of the difference of two successive iterates of the mechanics equation (14) and test by $\mathbf{v}_{h}=\partial_{t} \delta \mathbf{u}_{h}^{i}-\partial_{t} \delta \mathbf{u}_{h}^{i-1}$. By applying the Cauchy-Schwarz inequality, it follows

$$
\left\|\nabla \cdot\left(\partial_{t} \delta \mathbf{u}_{h}^{i}-\partial_{t} \delta \mathbf{u}_{h}^{i-1}\right)\right\| \leq \frac{\alpha}{\frac{2 G}{d}+\lambda}\left\|\partial_{t} \delta p_{h}^{i}-\partial_{t} \delta p_{h}^{i-1}\right\| .
$$

Inserting equality (16) into Eq. (19) and by applying Cauchy-Schwarz and (20) inequalities, we obtain

$$
\begin{aligned}
& \frac{G}{2}\left\|\boldsymbol{\varepsilon}\left(\partial_{t} \delta \mathbf{u}_{h}^{i}+\partial_{t} \delta \mathbf{u}_{h}^{i-1}\right)\right\|^{2}+\frac{\lambda}{4}\left\|\nabla \cdot\left(\partial_{t} \delta \mathbf{u}_{h}^{i}+\partial_{t} \delta \mathbf{u}_{h}^{i-1}\right)\right\|^{2}+\frac{1}{\beta}\left\|\partial_{t} \delta p_{h}^{i}\right\|^{2} \\
& +\frac{1}{2} \frac{d}{d t}\left\|\delta p_{h}^{i}\right\|_{B}^{2}+\frac{L}{2}\left(\left\|\partial_{t} \delta p_{h}^{i}\right\|^{2}+\left\|\partial_{t} \delta p_{h}^{i}-\partial_{t} \delta p_{h}^{i-1}\right\|^{2}\right) \\
& \leq \frac{L}{2}\left\|\partial_{t} \delta p_{h}^{i-1}\right\|^{2}+\frac{\alpha^{2}}{4\left(\frac{2 G}{d}+\lambda\right)}\left\|\partial_{t} \delta p_{h}^{i}-\partial_{t} \delta p_{h}^{i-1}\right\|^{2} .
\end{aligned}
$$

Discarding the first three positive terms, taking $L \geq \frac{\alpha^{2}}{2\left(\frac{2 G}{d}+\lambda\right)}$, and integrating from 0 to $t$ we finally obtain (15). It implies that the scheme is a contraction. Following the same technique as in [15], from the contractive property of scheme (13)-(14) one can establish that it is convergent and show that the converged quantities satisfy the variational formulation of the semi-discretized problem (9)-(10). This completes the proof.

Remark 2. It is easy to see that the fixed-stress splitting method in the semi-discrete case is an iterative method based on a suitable splitting for solving the differential/algebraic equation system (11). In detail, the iterative method can be written in the form

$$
\left[\begin{array}{cc}
0 & 0 \\
0 & (1+L) M_{p}
\end{array}\right]\left[\begin{array}{c}
\dot{u}_{h}^{i} \\
\dot{p}_{h}^{i}
\end{array}\right]+\left[\begin{array}{cc}
A & B^{t} \\
0 & -C
\end{array}\right]\left[\begin{array}{c}
u_{h}^{i} \\
p_{h}^{i}
\end{array}\right]=\left[\begin{array}{cc}
0 & 0 \\
-B & L M_{p}
\end{array}\right]\left[\begin{array}{c}
\dot{u}_{h}^{i-1} \\
\dot{p}_{h}^{i-1}
\end{array}\right]+\left[\begin{array}{c}
g_{h} \\
\widetilde{f}_{h}
\end{array}\right] .
$$

\section{The parallel-in-time fixed-stress splitting algorithm for the fully discretized problem}

\subsection{Parallel-in-time algorithm}

For time discretization we use the backward Euler method on a uniform partition $\left\{t_{0}, t_{1}, \ldots, t_{N}\right\}$ of the time interval $(0, T]$ with constant time-step size $\tau, N \tau=T$. Then, we have the following fully discrete scheme corresponding to (9)-(10): For $n=1,2, \ldots, N$, find $\left(\boldsymbol{u}_{h}^{n}, p_{h}^{n}\right) \in \boldsymbol{V}_{h} \times Q_{h}$ such that

$$
\begin{aligned}
& a\left(\boldsymbol{u}_{h}^{n}, \boldsymbol{v}_{h}\right)-\alpha\left(p_{h}^{n}, \operatorname{div} \boldsymbol{v}_{h}\right)=\left(\rho \mathbf{g}, \boldsymbol{v}_{h}\right), \quad \forall \boldsymbol{v}_{h} \in \boldsymbol{V}_{h}, \\
& \alpha\left(\operatorname{div} \bar{\partial}_{t} \boldsymbol{u}_{h}^{n}, q_{h}\right)+\frac{1}{\beta}\left(\bar{\partial}_{t} p_{h}^{n}, q_{h}\right)+b\left(p_{h}^{n}, q_{h}\right)=\left(f_{h}^{n}, q_{h}\right)+\left(\boldsymbol{K} \mu_{f}^{-1} \rho_{f} \mathbf{g}, \nabla q_{h}\right), \forall q_{h} \in Q_{h},
\end{aligned}
$$

where $\bar{\partial}_{t} \boldsymbol{u}_{h}^{n}:=\left(\boldsymbol{u}_{h}^{n}-\boldsymbol{u}_{h}^{n-1}\right) / \tau$ and $\bar{\partial}_{t} p_{h}^{n}:=\left(p_{h}^{n}-p_{h}^{n-1}\right) / \tau$.

We now discuss a partially parallel-in-time version of the fixed-stress splitting method. This algorithm arises in a natural way from the iterative method (21) by discretizing in time. In this way, given an initial guess $\left\{\left(\boldsymbol{u}_{h}^{n, 0}, p_{h}^{n, 0}\right), n=0,1, \ldots, N\right\}$, the new fixed-stress splitting algorithm gives us a sequence of approximations $\left\{\left(\boldsymbol{u}_{h}^{n, i}, p_{h}^{n, i}\right), n=0,1, \ldots, N\right\}, i \geq 1$, as follows:

Step 1: Let $p_{h}^{0, i}=p_{0}$, for all $i \geq 0$. For $i \geq 1$, given $\left\{\left(\boldsymbol{u}_{h}^{n, i-1}, p_{h}^{n, i-1}\right), n=0,1, \ldots, N\right\}$, find $p_{h}^{n, i} \in Q_{h}, n=1, \ldots, N$, such that $\forall q_{h} \in Q_{h}$ there holds

$$
\begin{gathered}
\left(\frac{1}{\beta}+L\right)\left(\frac{p_{h}^{n, i}-p_{h}^{n-1, i}}{\tau}, q_{h}\right)+b\left(p_{h}^{n, i}, q_{h}\right)=\alpha\left(\operatorname{div} \frac{\boldsymbol{u}_{h}^{n, i-1}-\boldsymbol{u}_{h}^{n-1, i-1}}{\tau}, q_{h}\right) \\
+L\left(\frac{p_{h}^{n, i-1}-p_{h}^{n-1, i-1}}{\tau}, q_{h}\right)+\left(f_{h}^{n}, q_{h}\right)+\left(\boldsymbol{K} \mu_{f}^{-1} \rho_{f} \mathbf{g}, \nabla q_{h}\right) .
\end{gathered}
$$

Step 2: Given $p_{h}^{n, i} \in Q_{h}, n=1, \ldots, N$, find $\boldsymbol{u}_{h}^{n, i} \in \boldsymbol{V}_{h}, n=1, \ldots, N$, such that

$$
a\left(\boldsymbol{u}_{h}^{n, i}, \boldsymbol{v}_{h}\right)=\alpha\left(p_{h}^{n, i}, \operatorname{div} \boldsymbol{v}_{h}\right)+\left(\rho \mathbf{g}, \boldsymbol{v}_{h}\right), \quad \forall \boldsymbol{v}_{h} \in \boldsymbol{V}_{h} .
$$


Remark 3. We wish to emphasize that the proposed method is partially parallel-in-time in contrast to the classical sequential fixed-stress splitting method based on time-stepping. Notice that in the Step 2 of the new algorithm, $N-1$ independent elliptic problems can be easily solved in parallel. Also the flow problem in Step 1 can be solved by using some of the wellknown parallel-in-time methods for parabolic problems mentioned in the introduction: PFASST, parareal, WRMG, MGRIT, or STMG. In this work, however, this step is implemented in a classical (non-parallel) form to keep the implementation simple, since the aim of this research is to present this new version of the fixed-stress iteration.

Remark 4 (A Fully Parallel-in-time Fixed-stress Scheme). The scheme (24)-(25) can be made fully parallel by replacing everywhere $p^{n-1, i}$ with $p^{n-1, i-1}$. The following scheme arises:

Step 1: Let $p_{h}^{0, i}=p_{0}$, for all $i \geq 0$. For $i \geq 1$, given $\left\{\left(\boldsymbol{u}_{h}^{n, i-1}, p_{h}^{n, i-1}\right), n=0,1, \ldots, N\right\}$, find $p_{h}^{n, i} \in Q_{h}, n=1, \ldots, N$, such that $\forall q_{h} \in Q_{h}$ there holds

$$
\begin{gathered}
\left(\frac{1}{\beta}+L\right)\left(\frac{p_{h}^{n, i}-p_{h}^{n-1, i-1}}{\tau}, q_{h}\right)+b\left(p_{h}^{n, i}, q_{h}\right)=\alpha\left(\operatorname{div} \frac{\boldsymbol{u}_{h}^{n, i-1}-\boldsymbol{u}_{h}^{n-1, i-1}}{\tau}, q_{h}\right) \\
+L\left(\frac{p_{h}^{n, i-1}-p_{h}^{n-1, i-1}}{\tau}, q_{h}\right)+\left(f_{h}^{n}, q_{h}\right)+\left(\boldsymbol{K} \mu_{f}^{-1} \rho_{f} \boldsymbol{g}, \nabla q_{h}\right) .
\end{gathered}
$$

Step 2: Given $p_{h}^{n, i} \in Q_{h}, n=1, \ldots, N$, find $\boldsymbol{u}_{h}^{n, i} \in \boldsymbol{V}_{h}, n=1, \ldots, N$, such that

$$
a\left(\boldsymbol{u}_{h}^{n, i}, \boldsymbol{v}_{h}\right)=\alpha\left(p_{h}^{n, i}, \operatorname{div} \boldsymbol{v}_{h}\right)+\left(\rho \mathbf{g}, \boldsymbol{v}_{h}\right), \quad \forall \boldsymbol{v}_{h} \in \boldsymbol{V}_{h} .
$$

The fully parallel-in-time scheme needs for the numerical examples considered more iterations (for the same tuning parameter) than the partially parallel scheme. A throughout analysis of this second scheme is beyond the aim of this paper.

\subsection{Convergence analysis of the partially parallel-in-time scheme}

Let $\delta \boldsymbol{u}_{h}^{n, i}=\boldsymbol{u}_{h}^{n, i}-\boldsymbol{u}_{h}^{n, i-1}$ and $\delta p_{h}^{n, i}=p_{h}^{n, i}-p_{h}^{n, i-1}$ denote the difference between two succesive approximations for displacements and for pressure, respectively.

Theorem 2. The fixed-stress splitting method given in (24)-(25) is convergent for any stabilization parameter $L \geq \frac{\alpha^{2}}{2\left(\frac{2 G}{d}+\lambda\right)}$. There holds

$$
\sum_{n=1}^{N} \tau\left\|\bar{\partial}_{t} \delta p_{h}^{n, i}\right\|^{2} \leq \frac{L}{\left(\frac{1}{\beta}+L\right)} \sum_{n=1}^{N} \tau\left\|\bar{\partial}_{t} \delta p_{h}^{n, i-1}\right\|^{2} .
$$

Proof. Similarly to the proof of Theorem 1, we take the difference of two successive iterates of the mechanics equation (25) and the flow equation (24), and test the resulting equations by $\mathbf{v}_{h}=\bar{\partial}_{t} \delta \mathbf{u}_{h}^{n, i-1}$ and $q_{h}=\bar{\partial}_{t} \delta p_{h}^{n, i}$ respectively to get for $n=1,2, \ldots, N$,

$$
\begin{aligned}
2 G\left(\boldsymbol{\varepsilon}\left(\bar{\partial}_{t} \delta \mathbf{u}_{h}^{n, i}\right), \boldsymbol{\varepsilon}\left(\bar{\partial}_{t} \delta \mathbf{u}_{h}^{n, i-1}\right)\right)+\lambda\left(\nabla \cdot \bar{\partial}_{t} \delta \mathbf{u}_{h}^{n, i}, \nabla \cdot \bar{\partial}_{t} \delta \mathbf{u}_{h}^{n, i-1}\right) & \\
- & \alpha\left(\bar{\partial}_{t} \delta p_{h}^{n, i}, \nabla \cdot \bar{\partial}_{t} \delta \mathbf{u}_{h}^{n, i-1}\right)=0 . \\
\frac{1}{\beta}\left\|\bar{\partial}_{t} \delta p_{h}^{n, i}\right\|^{2}+ & L\left(\bar{\partial}_{t}\left(\delta p_{h}^{n, i}-\delta p_{h}^{n, i-1}\right), \bar{\partial}_{t} \delta p_{h}^{n, i}\right)+b\left(\delta p_{h}^{n, i}, \bar{\partial}_{t} \delta p_{h}^{n, i}\right) \\
+ & \alpha\left(\nabla \cdot \bar{\partial}_{t} \delta \mathbf{u}_{h}^{n, i-1}, \bar{\partial}_{t} \delta p_{h}^{n, i}\right)=0 .
\end{aligned}
$$

After summing up Eqs. (29) and (30), and using the identities in (18) one has

$$
\begin{gathered}
\frac{G}{2}\left\|\boldsymbol{\varepsilon}\left(\bar{\partial}_{t} \delta \mathbf{u}_{h}^{n, i}+\bar{\partial}_{t} \delta \mathbf{u}_{h}^{n, i-1}\right)\right\|^{2}+\frac{\lambda}{4}\left\|\nabla \cdot\left(\bar{\partial}_{t} \delta \mathbf{u}_{h}^{n, i}+\bar{\partial}_{t} \delta \mathbf{u}_{h}^{n, i-1}\right)\right\|^{2}+\frac{1}{\beta}\left\|\bar{\partial}_{t} \delta p_{h}^{n, i}\right\|^{2} \\
+\frac{L}{2}\left(\left\|\bar{\partial}_{t} \delta p_{h}^{n, i}\right\|^{2}+\left\|\bar{\partial}_{t} \delta p_{h}^{n, i}-\bar{\partial}_{t} \delta p_{h}^{n, i-1}\right\|^{2}\right)+\frac{1}{2 \tau}\left(\left\|\delta p_{h}^{n, i}\right\|_{B}^{2}+\left\|\delta p_{h}^{n, i}-\delta p_{h}^{n-1, i}\right\|_{B}^{2}\right) \\
=\frac{G}{2}\left\|\boldsymbol{\varepsilon}\left(\bar{\partial}_{t} \delta \mathbf{u}_{h}^{n, i}-\bar{\partial}_{t} \delta \mathbf{u}_{h}^{n, i-1}\right)\right\|^{2}+\frac{\lambda}{4}\left\|\nabla \cdot\left(\bar{\partial}_{t} \delta \mathbf{u}_{h}^{n, i}-\bar{\partial}_{t} \delta \mathbf{u}_{h}^{n, i-1}\right)\right\|^{2} \\
+\frac{L}{2}\left\|\bar{\partial}_{t} \delta p_{h}^{n, i-1}\right\|^{2}+\frac{1}{2 \tau}\left\|\delta p_{h}^{n-1, i}\right\|_{B}^{2} .
\end{gathered}
$$


Next, we consider the difference of two successive iterates of the mechanics equation (25) and test by $\mathbf{v}_{h}=\bar{\partial}_{t} \delta \mathbf{u}_{h}^{n, i}-\bar{\partial}_{t} \delta \mathbf{u}_{h}^{n, i-1}$ to get

$$
\begin{aligned}
2 G\left\|\boldsymbol{\varepsilon}\left(\bar{\partial}_{t} \delta \mathbf{u}_{h}^{n, i}-\bar{\partial}_{t} \delta \mathbf{u}_{h}^{n, i-1}\right)\right\|^{2}+\lambda\left\|\nabla \cdot\left(\bar{\partial}_{t} \delta \mathbf{u}_{h}^{n, i}-\bar{\partial}_{t} \delta \mathbf{u}_{h}^{n, i}\right)\right\|^{2} \\
=\alpha\left(\bar{\partial}_{t} \delta p_{h}^{n, i}-\bar{\partial}_{t} \delta p_{h}^{n, i-1}, \nabla \cdot\left(\bar{\partial}_{t} \delta \mathbf{u}_{h}^{n, i}-\bar{\partial}_{t} \delta \mathbf{u}_{h}^{n, i-1}\right)\right) .
\end{aligned}
$$

From this equality, by applying Cauchy-Schwarz inequality, it is easy to see

$$
\left\|\nabla \cdot\left(\bar{\partial}_{t} \delta \mathbf{u}_{h}^{n, i}-\bar{\partial}_{t} \delta \mathbf{u}_{h}^{n, i-1}\right)\right\| \leq \frac{\alpha}{\frac{2 G}{d}+\lambda}\left\|\bar{\partial}_{t} \delta p_{h}^{n, i}-\bar{\partial}_{t} \delta p_{h}^{n, i-1}\right\| .
$$

Inserting equality (32) into Eq. (31) and by applying Cauchy-Schwarz inequality and (33), we obtain

$$
\begin{gathered}
\frac{G}{2}\left\|\boldsymbol{\varepsilon}\left(\bar{\partial}_{t} \delta \mathbf{u}_{h}^{n, i}+\bar{\partial}_{t} \delta \mathbf{u}_{h}^{n, i-1}\right)\right\|^{2}+\frac{\lambda}{4}\left\|\nabla \cdot\left(\bar{\partial}_{t} \delta \mathbf{u}_{h}^{n, i}+\bar{\partial}_{t} \delta \mathbf{u}_{h}^{n, i-1}\right)\right\|^{2}+\frac{1}{\beta}\left\|\bar{\partial}_{t} \delta p_{h}^{n, i}\right\|^{2} \\
+\frac{L}{2}\left(\left\|\bar{\partial}_{t} \delta p_{h}^{n, i}\right\|^{2}+\left\|\bar{\partial}_{t} \delta p_{h}^{n, i}-\bar{\partial}_{t} \delta p_{h}^{n, i-1}\right\|^{2}\right)+\frac{1}{2 \tau}\left(\left\|\delta p_{h}^{n, i}\right\|_{B}^{2}+\left\|\delta p_{h}^{n, i}-\delta p_{h}^{n-1, i}\right\|_{B}^{2}\right) \\
\leq \frac{L}{2}\left\|\bar{\partial}_{t} \delta p_{h}^{n, i-1}\right\|^{2}+\frac{1}{2 \tau}\left\|\delta p_{h}^{n-1, i}\right\|_{B}^{2}+\frac{\alpha^{2}}{4\left(\frac{2 G}{d}+\lambda\right)}\left\|\bar{\partial}_{t} \delta p_{h}^{n, i}-\bar{\partial}_{t} \delta p_{h}^{n, i-1}\right\|^{2} .
\end{gathered}
$$

Discarding positive terms, taking $L \geq \frac{\alpha^{2}}{2\left(\frac{2 G}{d}+\lambda\right)}$, and summing up from $n=1$ to $N$, we finally obtain (28). This implies that the scheme is a contraction and therefore convergent. This completes the proof.

Remark 5. Notice that the values of parameter $L$ turn out to be the same as in the classical fixed-stress splitting scheme.

\section{Numerical experiments}

In this section, we present two numerical experiments with the purpose of illustrating the performance of the partially parallel-in-time fixed-stress splitting (PFS) method described in Section 4. We compare the PFS method with the classical fixed-stress splitting (FS), see e.g. [14]. As first test problem, we use Mandel's problem, which is a well-established 2D benchmark problem with a known analytical solution [34,35]. This problem is very often used in the community for verifying the implementation and the performance of the numerical schemes, see e.g. $[5,10,30,36]$. As a second test, we use a threedimensional problem on a L-shaped domain with time dependent boundary conditions, see e.g. [16,37]. For both numerical experiments, a stabilized P1-P1 scheme has been used here for spatial discretization. However, we would like to mention that any stable pair could be considered instead.

The performance of both methods, FS and PFS, is similar if they are running sequentially. One of the main differences between the two methods, however, is the memory consumption. While FS uses a fixed memory amount independent to the time step, the PFS uses a memory amount proportional to the number of time steps. This is because PFS requires to store each variable for all time levels. However, to have access to the variables at every time step allows that several tasks of the implementation can run in parallel, mainly the solution of the mechanics problem at Eq. (25) for each time level and the assembly of the right hand sides at each time step.

The schemes were implemented in the open-source software package deal.II [38] configured for multithreading. The number of threads running in parallel simultaneously can be specified between 1 and 32 in a system of $4 \times 8$ cores Intel Xeon $2.7 \mathrm{GHz}$. In this regard, PFS is set to use one thread to solve the flow problem and up to 32 threads to assemble the right hand side, impose the boundary conditions, solve the mechanics problem and write the output results at each time step, while FS is set to use only one thread for the same tasks. In both numerical experiments, we report the absolute wall time of each method. We would like to mention that all the linear systems are solved by using a direct solver for simplicity in the implementation. However, a preconditioned conjugate gradient would be a good alternative for the efficient solution of such systems.

\subsection{Test case 1: Mandel's problem}

Mandel's problem consists of a poroelastic slab of extent $2 a$ in the $x$-direction, $2 b$ in the $y$-direction, and infinitely long in the $z$-direction, and is sandwiched between two rigid impermeable plates (see Fig. 1a). At time $t=0$, a uniform vertical load of magnitude $2 F$ is applied and an equal, but upward force is applied to the bottom plate. This load is supposed to remain constant. The domain is free to drain and stress-free at $x= \pm a$. Gravity is neglected.

For the numerical solution, the symmetry of the problem allows us to use a quarter of the physical domain as computational domain (see Fig. 1b). Moreover, the rigid plate condition is enforced by adding constrained equations so that vertical displacement $u_{y}(b, t)$ on the top is equal to a known constant value.

The application of a load $(2 F)$ causes an instantaneous and uniform pressure increase throughout the domain [39]; this is predicted theoretically [34] and it can be used as an initial condition 


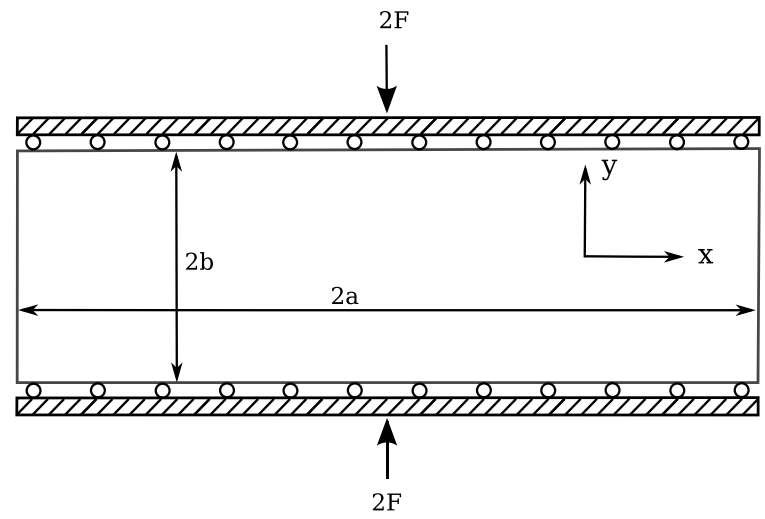

(a) Mandel's problem physical domain.

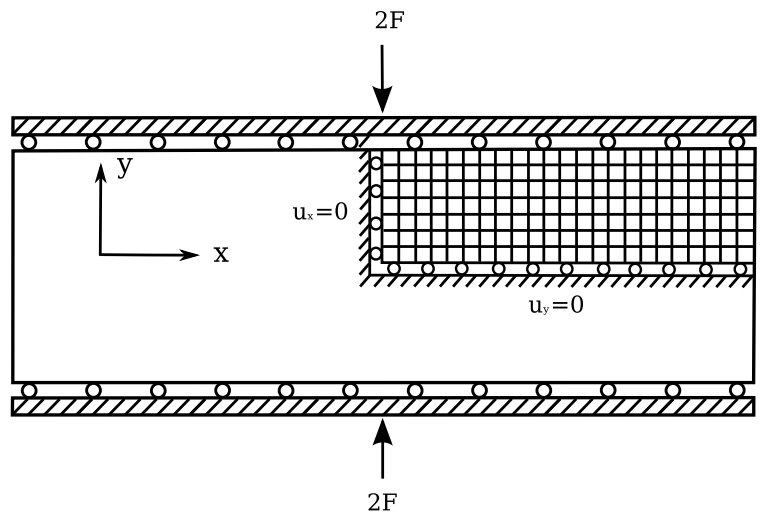

(b) Mandel's problem quarter domain.

Fig. 1. Mandel's problem.

Table 1

Boundary conditions for Mandel's problem.

\begin{tabular}{lll}
\hline Boundary & Flow & Mechanics \\
\hline$x=0$ & $\boldsymbol{q} \cdot \mathbf{n}=0$ & $\boldsymbol{u} \cdot \mathbf{n}=0$ \\
$y=0$ & $\mathbf{q} \cdot \mathbf{n}=0$ & $\boldsymbol{u} \cdot \mathbf{n}=0$ \\
$x=a$ & $p=0$ & $\sigma \cdot \mathbf{n}=0$ \\
$y=b$ & $\boldsymbol{q} \cdot \mathbf{n}=0$ & $\sigma_{12}=0 ; \boldsymbol{u} \cdot \mathbf{n}=u_{y}(b, t)$ \\
\hline
\end{tabular}

Table 2

Input parameter for Mandel's problem.

\begin{tabular}{llllll}
\hline Symbol & Quantity & Value & Symbol & Quantity & Value \\
\hline $\mathrm{a}$ & Dimension in $x$ & $100 \mathrm{~m}$ & $\mathrm{~b}$ & Dimension in $y$ & $10 \mathrm{~m}$ \\
$\mathrm{~K}$ & Permeability & $100 \mathrm{D}$ & $\mu_{f}$ & Dynamic viscosity & $10 \mathrm{cp}$ \\
$\alpha$ & Biot's constant & 1.0 & $\beta$ & Biot's modulus & $1.65 \times 10^{10} \mathrm{~Pa}$ \\
$v$ & Poisson's ratio & 0.4 & $E$ & Young's modulus & $5.94 \times 10^{9} \mathrm{~Pa}$ \\
$B$ & Skempton coefficient & 0.83333 & $v_{u}$ & Undrained Poisson's ratio & 0.44 \\
$c$ & Diffusivity coefficient & $46.526 \mathrm{~m}^{2} / \mathrm{s}$ & $F$ & Force intensity & $6.8 \times 10^{8} \mathrm{~N} / \mathrm{m}$ \\
$h_{x}$ & Grid spacing in $x$ & $2.5 \mathrm{~m}$ & $h_{y}$ & Grid spacing in $y$ & $h_{x} / 10$ \\
$\tau$ & Time step & $1 \mathrm{~s}$ & $T$ & Total simulation time & $32 \mathrm{~s}$ \\
\hline
\end{tabular}

$$
\begin{aligned}
p(x, y, 0) & =\frac{F B\left(1+v_{u}\right)}{3 a}, \\
\boldsymbol{u}(x, y, 0) & =\left(\frac{F v_{u} x}{2 G}, \quad \frac{-F b\left(1-v_{u}\right) y}{2 G a}\right)^{\top},
\end{aligned}
$$

where $B$ is the Skempton coefficient and $v_{u}=\frac{3 v+B(1-2 v)}{3-B(1-2 v)}$ is the undrained Poisson ratio.

The boundary conditions are specified in Table 1 and the input parameters for Mandel's problem are listed in Table 2. For all cases, the following stopping criterion is used $\left\|\delta p^{n, i}\right\|+\left\|\delta \boldsymbol{u}^{n, i}\right\| \leq 10^{-8}$.

In Fig. 2, the numerical and the analytical solutions of Mandel's problem are depicted for different values of time. There is a very good match between both solutions for all cases. Moreover, the results demonstrate the Mandel-Cryer effect, first showing a pressure raise during the first $20 \mathrm{~s}$ and then, a sudden dissipation throughout the domain.

The number of iterations for PFS and FS are reported in Fig. 3 for different values of parameter $L$ and various values of $v$. We remark a very similar behaviour of the two methods, with the optimal stabilization parameter $L$ being in this case the physical one $L_{p h y}:=\alpha^{2} /\left(\frac{2 G}{d}+\lambda\right)$, see e.g. $[10,13,14]$.

We remark that the mesh size and the time step $\tau$ do not influence the number of iterations. This can be seen in Table 3 , where we provide the number of iterations for both algorithms, varying the space and time discretization parameters.

Further, Fig. 4 shows the wall time for PFS reported for different mesh sizes and time steps (see Figs. 4a and 4b, respectively). The figure shows how the wall time decreases proportionally to the number of threads being used. However, the wall time does not decrease substantially when using more than 16 threads because of the sequential tasks that the code still has to perform (for instance solving the flow problem).

Table 4 shows the wall time for both FS and PFS. Since FS is running sequentially, it is set to use one thread. As expected, we clearly observe that PFS consumes around $20 \%$ of the wall time of FS. Furthermore, the more time steps are considered, the more this time reduction increases. 


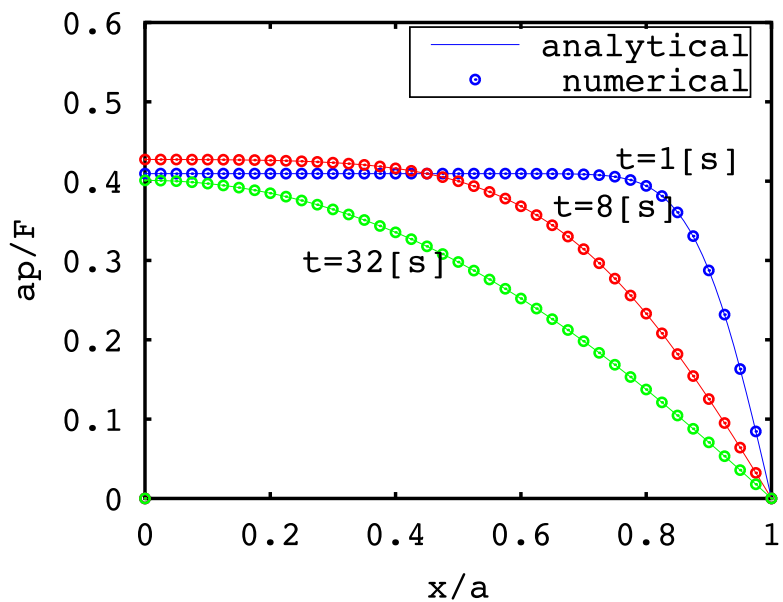

(a) Pressure solutions.

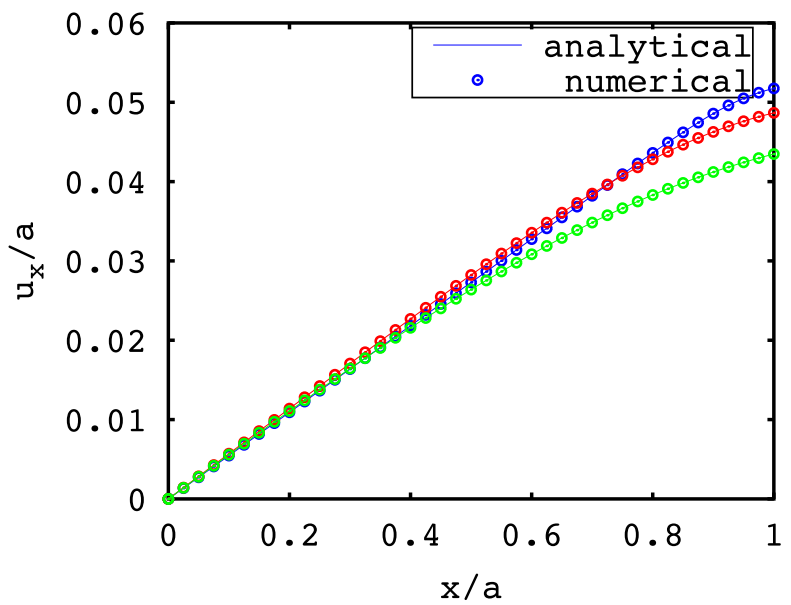

(b) Displacement solutions.

Fig. 2. Comparison of numerical and analytical solutions of the (a) pore pressure and (b) displacements for Mandel's problem in different times with $v=0.2$.

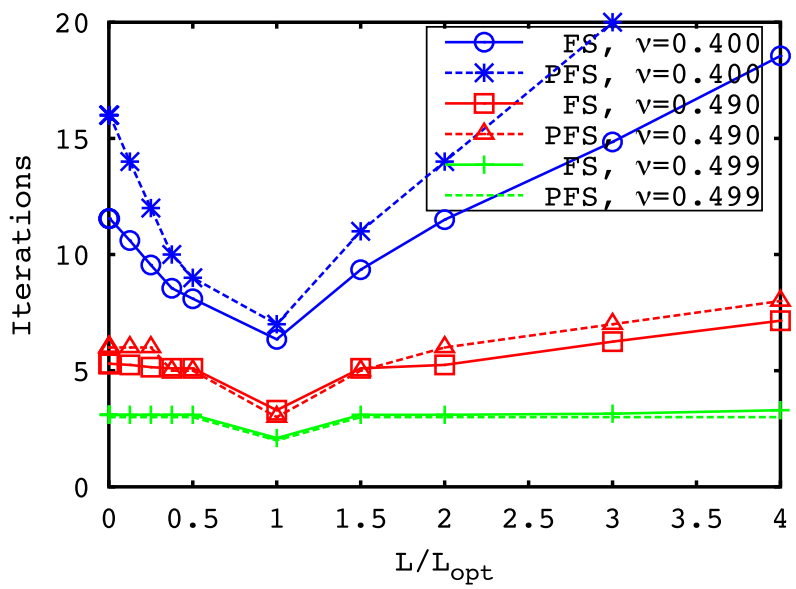

Fig. 3. Performance of the splitting schemes PFS and FS for different values of $L, \tau=1[s], h_{x}=2.5[\mathrm{~m}]$. Both schemes have the same optimum value $L_{\text {opt }}=L_{\text {phy }}$.

Table 3

Number of iterations for different values of $\tau, h_{x}, v$.

\begin{tabular}{lllllll}
\hline \multicolumn{2}{ll}{$v=0.49999}$, & $h_{x}=6.25[\mathrm{~m}]$. & & $v=0.499$, & \multicolumn{2}{l}{$\tau=0.5[\mathrm{~s}]}$. \\
\cline { 1 - 2 } \cline { 5 - 6 }$\tau[\mathrm{s}]$ & PFS & FS & & $h_{x}[\mathrm{~m}]$ & PFS & FS \\
\hline 1.000 & 2 & 2.10 & & 12.5000 & 3 & 3.20 \\
0.500 & 2 & 2.03 & & 6.2500 & 3 & 3.20 \\
0.250 & 2 & 2.02 & 3.1250 & 3 & 3.19 \\
0.125 & 2 & 2.01 & 1.5625 & 3 & 3.19 \\
\hline
\end{tabular}

\subsection{Test case 2: Poroelastic L-shaped problem}

The second numerical example is taken from [16,37]. It consists of a poroelastic L-shaped domain $\Omega \subset \mathbb{R}^{3}$ (see Fig. 5), with the long and short edges in the $x$ and $y$-direction being $1[\mathrm{~m}]$ and $0.5[\mathrm{~m}]$ respectively and an extrusion of $0.5[\mathrm{~m}]$ in the $z$-direction. The boundary conditions, numerical solution and input parameters are shown in Figs. 5-6 and Table 5. Gravity is neglected.

The number of iterations for the PFS method and the classical FS method are reported in Fig. 7 for different values of parameters $L$ and $v$. The methods show again a very similar behaviour. 


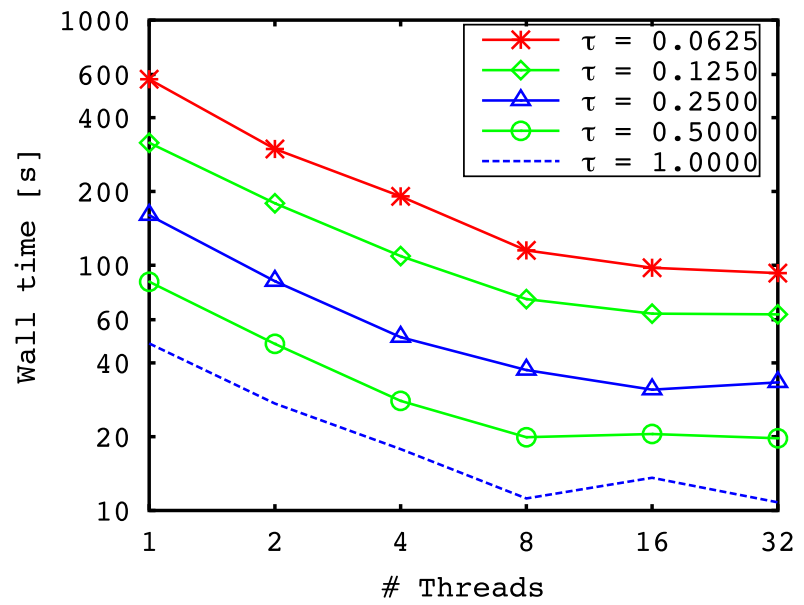

(a) Wall time for different time step sizes; $h_{x}=1.5625[m]$.

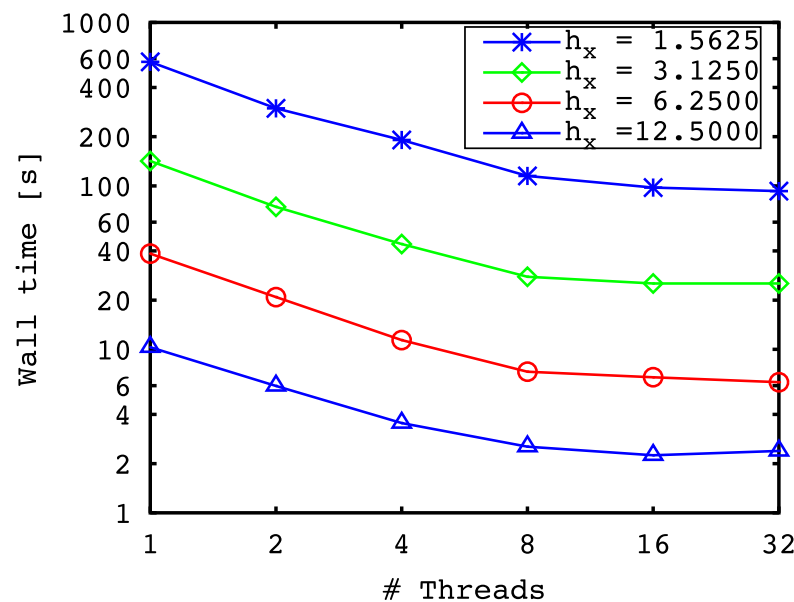

(b) Wall time for different mesh sizes; $\tau=0.0625[s]$.

Fig. 4. Wall time for different discretization parameters in space and time (first test).

Table 4

Wall time of FS/PFS for different time step sizes; $h_{x}=6.25[\mathrm{~m}]$.

\begin{tabular}{|c|c|c|c|c|c|c|}
\hline & $\tau[\mathrm{s}]$ & 1.0 & 0.5 & 0.25 & 0.125 & 0.0625 \\
\hline Method & \# Threads & \multicolumn{5}{|c|}{ Wall time [s] } \\
\hline FS & 1 & 43.3 & 78.6 & 146.0 & 281.0 & 499.0 \\
\hline \multirow[t]{6}{*}{ PFS } & 1 & 48.0 & 85.7 & 160.0 & 316.0 & 574.0 \\
\hline & 2 & 27.3 & 47.8 & 86.1 & 179.0 & 298.0 \\
\hline & 4 & 17.8 & 28.0 & 50.9 & 109.0 & 191.0 \\
\hline & 8 & 11.2 & 19.9 & 37.4 & 72.8 & 115.0 \\
\hline & 16 & 13.6 & 20.5 & 31.1 & 63.5 & 97.7 \\
\hline & 32 & 10.8 & 19.7 & 33.3 & 63.1 & 92.9 \\
\hline
\end{tabular}

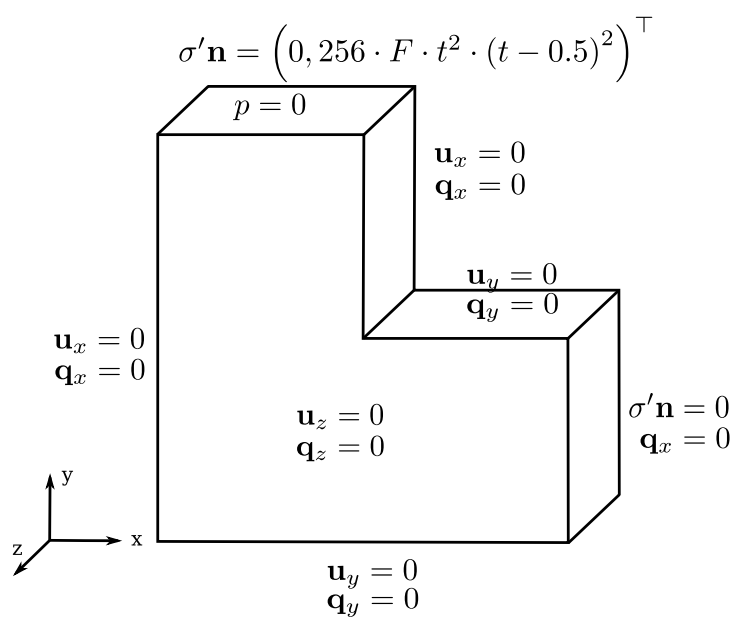

Fig. 5. L-shaped domain and boundary conditions (symmetric in the $z$-direction).

We remark again that the mesh size and the time step do not influence the number of iterations. This can be seen in Table 6, where we provide the number of iterations for both algorithms, varying the space and time discretization parameters.

Fig. 8 shows the wall time of PFS for different time steps and mesh sizes. Again, we observe that as the number of time steps increases the more threads are used the more the PFS reduces the wall time. In Table 7 we report the wall time of FS vs. PFS, observing a similar behaviour as in the first experiment. 

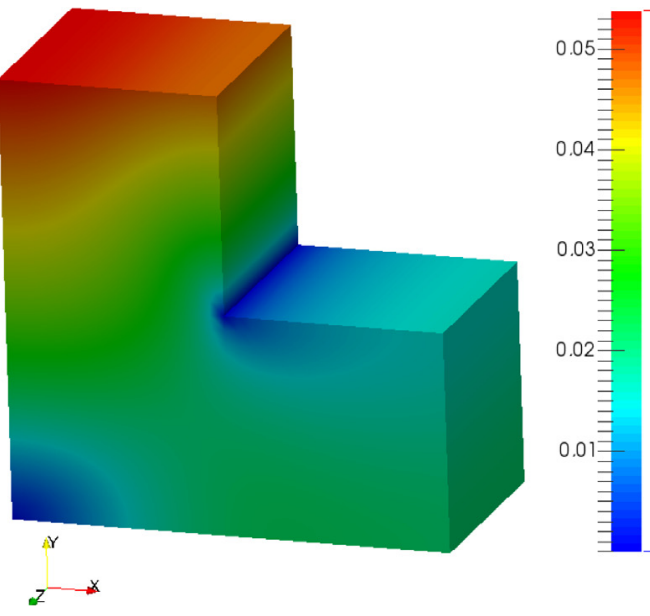

(a) Displacement's magnitude $[\mathrm{m}]$.

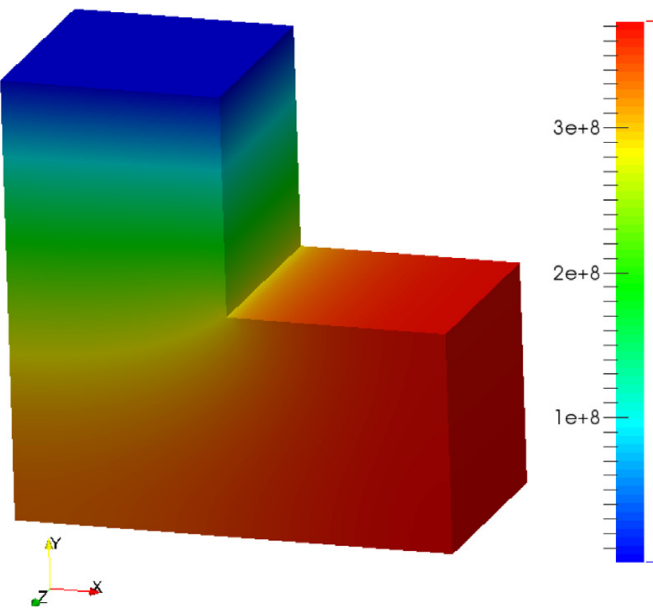

(b) Pressure $[\mathrm{Pa}]$.

Fig. 6. Numerical solutions at $t=0.26$ and $v=0.4, L=L_{\text {phy }}$.

Table 5

Input parameters for L-shaped problem.

\begin{tabular}{llllll}
\hline Symbol & Quantity & Value & Symbol & Quantity & Value \\
\hline $\mathrm{K}$ & Permeability & $1000 \mathrm{mD}$ & $\mu_{f}$ & Dynamic viscosity & $10 \mathrm{cp}$ \\
$\alpha$ & Biot's constant & 0.9 & $\beta$ & Biot's modulus & $100 \times 10^{9} \mathrm{~Pa}$ \\
$\nu$ & Poisson's ratio & 0.4 & $E$ & Young's modulus & $100 \times 10^{9} \mathrm{~Pa}$ \\
$h$ & Grid spacing & $1 / 2^{5} \mathrm{~m}$ & $F$ & Traction force constant & $10 \times 10^{9} \mathrm{~Pa}$ \\
$\tau$ & Time step & $0.01 \mathrm{~s}$ & $T$ & Total simulation time & $0.5 \mathrm{~s}$ \\
\hline
\end{tabular}

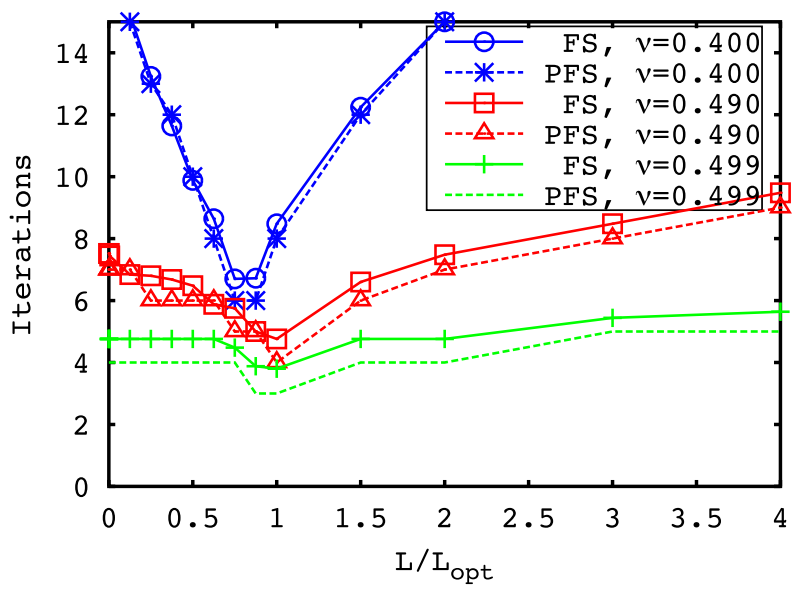

Fig. 7. Performance of the splitting schemes PFS and FS for different values of $L, \tau=0.02[\mathrm{~s}], h=0.03125[\mathrm{~m}], L_{\text {opt }}=\frac{L_{p h y}}{2}$.

Table 6

Number of iterations for different values of $\tau, h, v$.

\begin{tabular}{|c|c|c|c|c|c|}
\hline \multicolumn{3}{|c|}{$v=0.49999, h=0.125[\mathrm{~m}]}$. & \multicolumn{3}{|c|}{$v=0.499, \tau=0.02[\mathrm{~s}]}$. \\
\hline$\tau[\mathrm{s}]$ & PFS & FS & $h[\mathrm{~m}]$ & PFS & FS \\
\hline 0.050 & 2 & 2.80 & 0.25000 & 3 & 3.82 \\
\hline 0.020 & 2 & 2.84 & 0.12500 & 3 & 3.80 \\
\hline 0.010 & 2 & 2.94 & 0.06250 & 3 & 3.78 \\
\hline 0.005 & 2 & 2.97 & 0.03125 & 3 & 3.78 \\
\hline
\end{tabular}




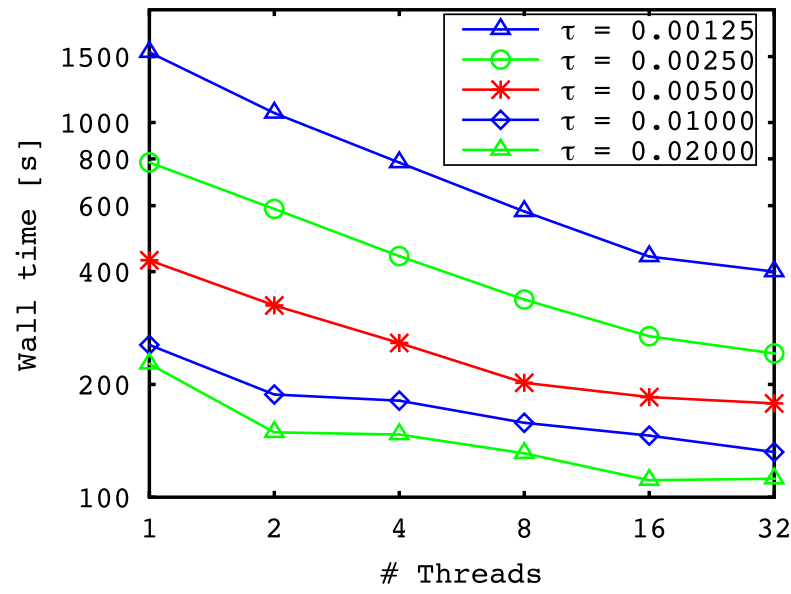

(a) Wall time for different time step sizes; $h=0.03125[m]$.

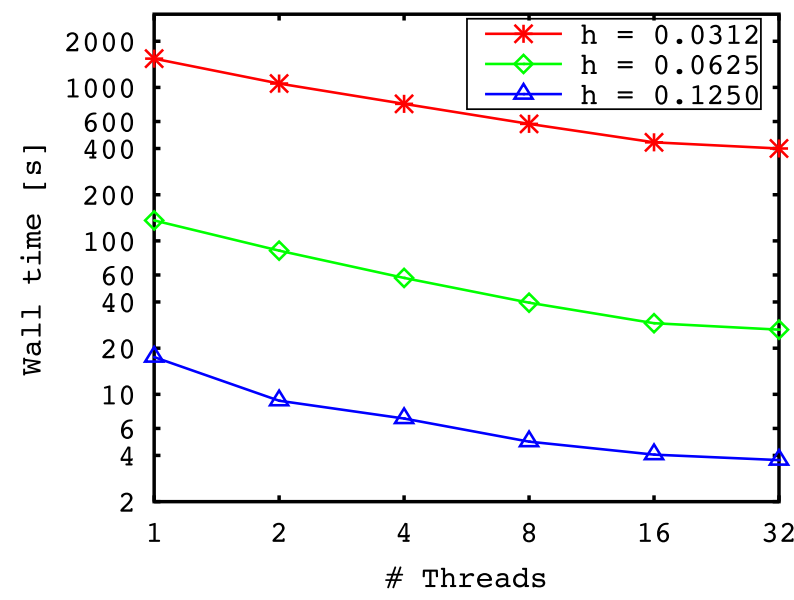

(b) Wall time for different time step sizes; $\tau=0.00125[m]$.

Fig. 8. Wall time for different discretization parameters in space and time (second test).

Table 7

Wall time in seconds of FS/PFS for different time step sizes; $h=0.0625[\mathrm{~m}]$.

\begin{tabular}{lllllll}
\hline & $\tau[\mathrm{s}]$ & 0.02 & 0.01 & 0.005 & 0.0025 & 0.00125 \\
\hline Method & \# Trheats & Wall time [s] & & & & \\
PFS & 1 & 10.6 & 17.1 & 31.2 & 63.6 & 115.0 \\
& 1 & 11.5 & 19.2 & 35.5 & 68.7 & 136.0 \\
& 2 & 8.5 & 12.1 & 22.3 & 44.1 & 86.4 \\
& 4 & 7.2 & 11.7 & 17.9 & 30.2 & 57.4 \\
& 8 & 7.1 & 8.7 & 14.7 & 21.6 & 39.6 \\
& 16 & 7.7 & 7.6 & 11.9 & 19.3 & 29.1 \\
\hline
\end{tabular}

\section{Conclusions}

We considered the quasi-static Biot model in the two-field formulation and presented a new fixed-stress type splitting method for solving it. The main benefit of the new method is that the mechanics can be solved in a parallel-in-time manner. We have rigorously analysed the convergence of the proposed method. If the stabilization term $L$ is chosen big enough, the method is shown to be convergent. The theoretical results are indicating a similar behaviour with the classical fixed-stress splitting method (in terms of convergence rate and stabilization parameter size). We further performed numerical tests by using two well-known benchmark problems. The numerical results confirm the theoretical findings. We observe that the new scheme PFS is very efficient (around $20 \%$ of the wall time of FS). Nevertheless, the parallel implementation has still to be optimized. A combination of the new scheme with a parallel algorithm for solving the flow (like e.g. PFASST, parareal, WRMG, MGRIT, or STMG) would substantially increase the efficiency.

\section{Acknowledgments}

The work of F.A. Radu and K. Kumar was partially supported by the NFR - Toppforsk project TheMSES, Norway, \#250223 and Norwegian Academy of Science and Equinor, Norway, through VISTA AdaSim no. 6367. The work of F.J. Gaspar is supported by the European Union's Horizon 2020 research and innovation programme, Spain, under the Marie SklodowskaCurie grant agreement NO 705402, POROSOS. The research of C. Rodrigo is supported in part by the Spanish project FEDER /MCYT MTM2016-75139-R and the DGA, Spain (Grupo de Referencia APEDIF, ref. E24_17R). We also want to thank the anonymous reviewers for helping us to substantially improve the paper and Dr. Uwe Köcher for his help concerning parallel computing and deal.II.

\section{References}

[1] K. Terzaghi, Theoretical Soil Mechanics, Wiley, New York, 1943, http://dx.doi.org/10.1002/9780470172766.

[2] M.A. Biot, General theory of three-dimensional consolidation, J. Appl. Phys. 12 (2) (1941) 155-164, http://dx.doi.org/10.1063/1.1712886 
[3] M.A. Biot, Theory of elasticity and consolidation for a porous anisotropic solid, J. Appl. Phys. 26 (2) (1955) 182-185, http://dx.doi.org/10.1063/1. 1721956.

[4] R. Showalter, Diffusion in poro-elastic media, J. Math. Anal. Appl. 251 (1) (2000) 310-340, http://dx.doi.org/10.1006/jmaa.2000.7048.

[5] P. Phillips, M. Wheeler, A coupling of mixed and continuous Galerkin finite element methods for poroelasticity I: the continuous in time case, Comput. Geosci. 11 (2) (2007) 131-144, http://dx.doi.org/10.1007/s10596-007-9045-y.

[6] L. Bergamaschi, M. Ferronato, G. Gambolati, Novel preconditioners for the iterative solution to FE-discretized coupled consolidation equations, Comput. Methods Appl. Mech. Eng. 196 (25) (2007) 2647-2656, http://dx.doi.org/10.1016/j.cma.2007.01.013.

[7] M. Ferronato, L. Bergamaschi, G. Gambolati, Performance and robustness of block constraint preconditioners in finite element coupled consolidation problems, Internat. J. Numer. Methods Engrg. 81 (2010) 381-402, http://dx.doi.org/10.1002/nla.372.

[8] F.J. Gaspar, F.J. Lisbona, C. Oosterlee, R. Wienands, A systematic comparison of coupled and distributive smoothing in multigrid for the poroelasticity system, Numer. Linear Algebra Appl. 11 (2004) 93-113, http://dx.doi.org/10.1002/nla.372.

[9] P. Luo, C. Rodrigo, F.J. Gaspar, C.W. Oosterlee, On an Uzawa smoother in multigrid for poroelasticity equations, Numer. Linear Algebra Appl. 24 (1) (2017) http://dx.doi.org/10.1002/nla.2074.

[10] J. Kim, H.A. Tchelepi, R. Juanes, Stability, accuracy and efficiency of sequential methods for coupled flow and geomechanics, in: The SPE Reservoir Simulation Symposium, Houston, Texas, 2009 SPE 119084. http://dx.doi.org/10.2118/119084-PA.

[11] J. Kim, H.A. Tchelepi, R. Juanes, Stability and convergence of sequential methods for coupled flow and geomechanics: fixed-stress and fixed-strain splits, Comput. Methods Appl. Mech. Eng. 200 (13-16) (2011) 1591-1606, http://dx.doi.org/10.1016/j.cma.2011.02.011.

[12] J. Kim, H.A. Tchelepi, R. Juanes, Stability and convergence of sequential methods for coupled flow and geomechanics: Drained and undrained splits, Comput. Methods Appl. Mech. Eng. 200 (23-24) (2011) 2094-2116, http://dx.doi.org/10.1016/j.cma.2010.12.022.

[13] A. Mikelić, M.F. Wheeler, Convergence of iterative coupling for coupled flow and geomechanics, Comput. Geosci. (17) (2013) 455-461, http://dx.doi. org/10.1007/s10596-012-9318-y.

[14] J.W. Both, M. Borregales, J.M. Nordbotten, K. Kumar, F.A. Radu, Robust fixed stress splitting for Biot's equations in heterogeneous media, Appl. Math. Lett. 68 (2017) 101-108, http://dx.doi.org/10.1016/j.aml.2016.12.019.

[15] T. Almani, K. Kumar, A. Dogru, G. Singh, M.F. Wheeler, Convergence analysis of multirate fixed-stress split iterative schemes for coupling flow with geomechanics, Comput. Methods Appl. Mech. Eng. 311 (1) (2016) 180-207, http://dx.doi.org/10.1016/j.cma.2016.07.036.

[16] M. Bause, F.A. Radu, U. Köcher, Space-time finite element approximation of the Biot poroelasticity system with iterative coupling, Comput. Methods Appl. Mech. Eng. 320 (2017) 745-768, http://dx.doi.org/10.1016/j.cma.2017.03.017.

[17] N. Castelleto, J.A. White, H.A. Tchelepi, Accuracy and convergence properties of the fixed-stress iterative solution of two-way coupled poromechanics, Int. J. Numer. Anal. Methods Geomech. 39 (2015) 1593-1618, http://dx.doi.org/10.1002/nag.2400.

[18] F.J. Gaspar, C. Rodrigo, On the fixed-stress split scheme as smoother in multigrid methods for coupling flow and geomechanics, Comput. Methods Appl. Mech. Eng. 326 (2017) 526-540, http://dx.doi.org/10.1016/j.cma.2017.08.025.

[19] M. Borregales, F.A. Radu, K. Kumar, J.M. Nordbotten, Robust iterative schemes for non-linear poromechanics, Comput. Geosci. 22 (2018) 1021-1038, http://dx.doi.org/10.1007/s10596-018-9736-6.

[20] J.W. Both, J.M. Nordbotten, K. Kumar, F.A. Radu, Anderson accelerated fixed-stress splitting schemes for consolidation of unsaturated porous media, Comput. Math. Appl. (2018) 1-24, http://dx.doi.org/10.1016/j.camwa.2018.07.033.

[21] M.J. Gander, 50 Years of Time Parallel Time Integration, Springer International Publishing, Cham, 2015, pp. 69-113, http://dx.doi.org/10.1007/9783-319-23321-5_3.

[22] M. Emmett, M.L. Minion, Toward an efficient parallel in time method for partial differential equations, Commun. Appl. Math. Comput. Sci. 7 (2012) 105-132, http://dx.doi.org/10.2140/camcos.2012.7.105.

[23] J. Lions, Y. Maday, G.A. Turinici, A “parareal” in time discretization of PDE's, C.R. Math. Acad. Sci. Ser. I Math., 332, 661-668. http://dx.doi.org/10.1016/ S0764-4442(00)01793-6.

[24] R.D. Falgout, S. Friedhoff, T.V. Kolev, S.P. MacLachlan, J.B. Schroder, Parallel time integration with multigrid, SIAM J. Sci. Comput. 16 (2014) 635-661, http://dx.doi.org/10.1137/130944230.

[25] G. Horton, S. Vandewalle, A space-time multigrid method for parabolic partial differential equations, SIAM J. Sci. Comput. 16 (4) (1995) 848-864, http://dx.doi.org/10.1137/0916050.

[26] C. Lubich, A. Ostermann, Multi-grid dynamic iteration for parabolic equations, BIT Numer. Math. 27 (2) (1987) 216-234, http://dx.doi.org/10.1007/ BF01934186.

[27] G. Horton, S. Vandewalle, P. Worley, An algorithm with polylog parallel complexity for solving parabolic partial differential equations, SIAM J. Sci. Comput. 16 (3) (1995) 531-541, http://dx.doi.org/10.1137/0916034.

[28] A. Ženíšek, The existence and uniqueness theorem in Biot's consolidation theory, Apl. Mat. 29 (3) (1984) 194-211.

[29] G. Aguilar, F. Gaspar, F. Lisbona, C. Rodrigo, Numerical stabilization of Biot's consolidation model by a perturbation on the flow equation, Int. J. Numer. Methods Eng. 75 (11) (2008) 1282-1300, http://dx.doi.org/10.1002/nme.2295.

[30] C. Rodrigo, F. Gaspar, X. Hu, L. Zikatanov, Stability and monotonicity for some discretizations of the Biot's consolidation model, Comput. Methods Appl. Mech. Eng. 298 (2016) 183-204, http://dx.doi.org/10.1016/j.cma.2015.09.019.

[31] M.A. Murad, A.F.D. Loula, Improved accuracy in finite element analysis of Biot's consolidation problem, Comput. Methods Appl. Mech. Engrg. 95 (3) (1992) 359-382, http://dx.doi.org/10.1016/0045-7825(92)90193-N.

[32] M.A. Murad, A.F.D. Loula, On stability and convergence of finite element approximations of Biot's consolidation problem, Internat. J. Numer. Methods Engrg. 37 (4) (1994) 645-667, http://dx.doi.org/10.1002/nme.1620370407.

[33] M.A. Murad, V. Thomée, A.F.D. Loula, Asymptotic behavior of semidiscrete finite-element approximations of Biot's consolidation problem, SIAM J. Numer. Anal. 33 (3) (1996) 1065-1083, http://dx.doi.org/10.1137/0733052.

[34] Y. Abousleiman, A.-D. Cheng, L. Cui, E. Detournay, J.-C. Roegiers, Mandel's problem revisited, Geotechnique 46 (2) (1996) 187-195, http://dx.doi.org/ 10.1680/geot.1996.46.2.187.

[35] J. Mandel, Consolidation Des Sols (étude de mathématique), Géotechnique 3 (1953) 287-299, http://dx.doi.org/10.1680/geot.1953.3.7.287.

[36] A. Mikelić, B. Wang, M.F. Wheeler, Numerical convergence study of iterative coupling for coupled flow and geomechanics, Comput. Geosci. 18 (3-4) (2014) 325-341, http://dx.doi.org/10.1007/s10596-013-9393-8.

[37] J.W. Both, U. Köcher, Numerical investigation on the fixed-stress split- ting scheme for Biots equations: Optimality of the tuning parameter, arXivPreprint arXiv:1801.08352.

[38] W. Bangerth, G. Kanschat, T. Heister, L. Heltai, G. Kanschat, The deal.II library version 8.4, J. Numer. Math. 24 (2016) 135-141, http://dx.doi.org/10. 1515/jnma-2016-1045.

[39] X. Gai, R.H. Dean, M.F. Wheeler, R. Liu, Coupled geomechanical and reservoir modeling on parallel computers, in: The SPE Reservoir Simulation Symposium, Houston, Texas, Feb. 3-5, 2003. http://dx.doi.org/10.2118/79700-MS. 